\title{
A Track Scoring Function Development for Airborne Target Detection Using Dynamic Programming
}

\author{
Dae-Yeon Won*, Sang-Wook Shim*, Keum-Seong Kim* and Min-Jea Tahk** \\ Korea Advanced Institute of Science and Technology, Daejeon, Republic of Korea
}

Eung-Tai Kim***

Korea Aerospace Research Institute, Daejeon, Republic of Korea

\begin{abstract}
Track-before-detect techniques based on dynamic programming have provided solutions for detecting targets from a sequence of images. In its application to airborne threat detection, dynamic programming solutions should take into account the distinguishable properties of objects in a collision course. This paper describes the development of a new track scoring function that accumulates scores for airborne targets in Bayesian framework. Numerical results show that the proposed scoring function has slightly better detection capabilities.
\end{abstract}

Key words: Dynamic programming, Airborne target detection, Track-before-detect

\section{Introduction}

Sense and avoid is one of the most basic responsibilities for aircraft operations. Pilots who operate aircraft are always required to maintain a high level of situation awareness in order to avoid a mid-air collision. Increased traffic densities and large speed differentials in airspace can have high potentials for air collisions even in daylight and in visual flight rule conditions. Thus, an autonomous collision detection and avoidance capability is a critical capability for a variety of unmanned aerial vehicle applications in airspace. In order to aid pilots or autonomous flight computers much effort has been made in developing robust collision detection algorithms. Recent improvements in the field of image processing and computer vision have raised interest in the use of image sequences from passive optical sensors for detecting potential threats [1-5].

Most of the studies have been carried out by using a track-before-detect (TBD) technique and this technique, integrates the sensor data about a tentative target over time by combining target detection and estimation. Unlike other conventional detection techniques such as probabilistic data association (PDA) and multiple hypotheses testing (MHT), a signal is tracked before declaring it as a target. In the literature, TBD methods based on dynamic programming (DP) have been proposed for dim target detection and tracking in highly cluttered environments where the signal-to-ratio (SNR) is relatively low [1-6]. The DP-based TBD methods effectively integrate the observations along possible trajectories and those trajectories for which the tracking score exceeds a given threshold are returned as possible targets [6]. The DP solution proposed by Barniv is one of the first papers in the literature [1] that has provided a fundamental approach to detect and track dim moving targets simultaneously as registered in an image set. Arnold et al. [3] improved the existing solutions in terms of computational efficiency and performance against nonGaussian noise characteristics. The analysis of the DP-based TBD was carried out by using extreme value theory in [6]. On the other hand, Nichtern et al. [7] dealt with some practical issues on setting the parameters of dynamic programming solutions in order to maximize the tracking capability. Although, there have been efforts to solve the various
This is an Open Access article distributed under the terms of the Creative Commons Attribution Non-Commercial License (http://creativecommons.org/licenses/by$\mathrm{nc} / 3.0 /$ ) which permits unrestricted non-commercial use, distribution and reproduction in any medium, provided the original work is properly cited.

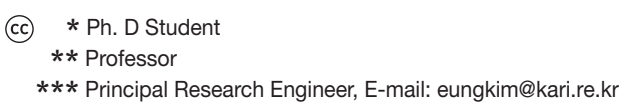


important TBD problems, the problem of developing track scoring functions has received less attention, particularly when it applies to airborne target detection on the flight path. As collision threats have a few geometric features from their relative dynamics, incorporating these features into track scoring functions is important to enhance the detection performance.

Thefundamentaltechniquepresented in this paperis based on the DP solutions that were proposed in $[1,3]$. However, our current work has led to some technical innovations in its application to the problem of airborne collision threat detection. In particular, a new track scoring function is proposed to improve the collision detection performance. This is done by reflecting two distinguishable properties of a non-maneuvering target in a collision course. First, a target on a near-collision course remains stationary in image view as collision occurs when the line-of-sight between aircrafts is maintained almost stationary. Second, the rate of image expansion for a collision course target is approximately inversely proportional to the time to collision [8]. As a result, the main problem of concern is in the development of a DP-based TBD technique that takes in to account these two properties for early airborne target detection.

This paper is organized as follows. In Section 2, the basic approach to DP-based TBD is described. Section 3 illustrates the development and implementation of a track score function of a DP solution for airborne target detection. Section 4 presents the numerical results and its performance assessment of the proposed method. The final section summarizes the overall discussion.

\section{Dynamic Programming Solutions for Air- borne Target Detection}

The objective of airborne target detection is to determine the trajectories that are most likely to have originated from the actual threat by using the measured image sequence of $K$ frames. Solutions for this problem should be able to provide high detection performance. Here, the size of threats can vary from sub-pixel to a few pixels in the presence of a background clutter. This section describes the outline of the DP algorithm and its application to solve TBD problem.

\subsection{Overall procedure}

The DP-based target detection algorithm has been divided into three steps. In the first step, the raw image sequence passes through pre-processing stages. It emphasizes the potential targets and provides a four-dimensional target intensity measurement set with the spatial and velocity resolution cells. Spatial resolution corresponds to the image sensor resolution. The velocity resolution depends on the design parameter which is related to a three-dimensional data processing stage. In this step, a close-minus-open filter which is one of the morphological filtering algorithms is employed to extract peak regions in images and to generate a non-negative output [9]. The second step is the DP-based target detection stage that operates on the unthresholded data set that is provided by the pre-processing step. Basically, the DP approach provides a single optimization procedure that includes both data association and track detection as it avoids a thresholding process. Moreover, it also preserves all the signal information in the raw image data. The DP-based TBD method presented hereafter is based on the approaches that are described in $[1,3]$. The final decision on the target presence and position is made in the third step by evaluating the criteria for both threshold values and uniqueness of track histories over a certain length of image frames.

\subsection{Target and Sensor Models}

Target motion is modeled to be approximately linear over the image plane of an image sensor, with allowances for curvature, acceleration, and jitter. This model illustrates the target motion onto the image plane with the resultant target dynamics and it described two differential equations of motion as shown in Fig. 1. The target model is defined by a discrete-time state-space equation of the form,

$$
\mathbf{x}_{k}=\mathbf{F}_{k} \mathbf{x}_{k-1}+\mathbf{G}_{k} \mathbf{u}_{k}
$$

Where, $\mathbf{x}_{k}=[x, y, u, v]_{k}^{T}$ is the target state vector at frame $k$. $(x, y) \in[(1,1),(L, L)]$ and $(u, v) \in[(-M / 2,-M / 2),(M / 2$, $M / 2)]$ are the horizontal and vertical relative target positions and velocities in discrete image dimensions, respectively. It is assumed that the discrete states are uniformly sampled over their ranges. The target state innovations are modeled as zero-mean random processes of the form,

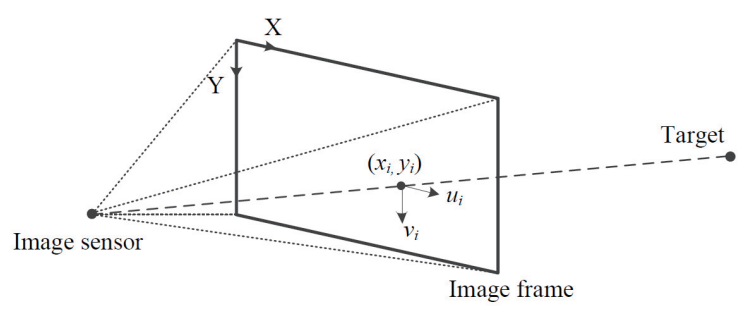

Fig. 1. Coordinate system for the target and sensor models. The relative target motion is defined by a discrete-time state-space equation on the image plane. 


$$
\mathbf{u}_{k}=\left[\zeta_{x_{k}}, \zeta_{y_{k}}, \dot{u}, \dot{v}\right]_{k}^{T}
$$

where $\zeta_{x_{k}}$ and $\zeta_{y_{k}}$ are the unpredictable target position transitions by sensor registration errors. $\dot{u}$ and $\dot{v}$ are the unknown target acceleration components. The process noises are zero-mean Gaussian white noises with unknown statistics. The state transition matrix, $\mathbf{F}_{k}$ and $\mathbf{G}_{k}$ are defined as follows by considering the problem of tracking a point target that moves with constant velocity in the image plane.

$$
\mathbf{F}_{k}=\left[\begin{array}{cccc}
1 & 0 & \Delta t_{k} & 0 \\
0 & 1 & 0 & \Delta t_{k} \\
0 & 0 & 1 & 0 \\
0 & 0 & 0 & 1
\end{array}\right], \mathbf{G}_{k}=\left[\begin{array}{cccc}
1 & 0 & 0.5 \Delta t_{k}^{2} & 0 \\
0 & 1 & 0 & 0.5 \Delta t_{k}^{2} \\
0 & 0 & \Delta t_{k} & 0 \\
0 & 0 & 0 & \Delta t_{k}
\end{array}\right] \text { (3) }
$$

where $\Delta t_{k}$ is the time interval between the $(k-1)$ th and the $k$ th image frames.

The raw image data are pre-processed to suppress clutter signals prior to the dynamic programming stage. The threedimensional convolution filter is applied to brief image sequences for the extraction of the target's expansion rate as well. Output from the image pre-processing step consists of discrete, four-dimensional matrices, $Z_{k}=\left\{\mathbf{q}_{k}, \mathbf{r}_{k}\right\} . \mathbf{q}_{k}$ and $\mathbf{r}_{k}$ are the amplitude and the expansion rate measurements of the target, respectively. At time $k$, each pixel of the measurement is modeled as follows,

$$
z_{k}^{(i, j)}=\left\{\begin{array}{cc}
h_{k}^{(i, j)}\left(\mathbf{x}_{k}\right)+w_{k}^{(i, j)} & , \text { target is present } \\
n_{k}^{(i, j)} & , \text { noise only }
\end{array}\right.
$$

where $h_{k}^{(i, j)}$ is the contribution of the target on each pixel and it relies on the point spread function and the state. $w_{k}$ is an additive noise with known statistics and $n_{k}$ is a sample from a random noise process. The noise distributions of $w_{k}$ and $n_{k}$ are generally independent and they are derived from different physical processes. As the rate of the target expansion is almost inversely proportional to the relative distance, $\mathbf{q}_{k}$ can be defined as a state if the state vector includes three-dimensional positions.

\subsection{Dynamic Programming Solution}

As the target state is assumed as the Markov process, the result of each stage depends only on the results of the previous stage. The maximum possible value of the scoring function for collision threat is obtained by the inclusion of a sequence of intermediate functions that represent the maximum partial sum of the previous scoring functions. Based on the posterior odds formulation, the scoring function, $s$ is defined as the logarithm of the ratio of posterior probabilities

$$
s\left(\mathbf{x}_{k}, \mathbf{x}_{k-1}, \ldots, \mathbf{x}_{1}\right)=\log \left[\frac{P_{k \mid k}\left(\mathbf{x}_{k}, \mathbf{x}_{k-1}, \ldots, \mathbf{x}_{1} \mid Z_{k}\right)}{P_{k \mid k}\left(H_{0} \mid Z_{k}\right)}\right]
$$

where $\left(\mathbf{x}_{k}, \mathbf{x}_{k-1}, \ldots, \mathbf{x}_{1}\right)$ is the target-present hypothesis for the specific target state sequence that enables in state $\mathbf{x}_{k}$ at time index $k$. The alternative hypothesis is represented by, $H_{0}$ while $Z_{k}$ represents the observation set. In the form of a DP solution such a procedure can be represented by a recursive form by resembling the following equation.

$$
h_{k}\left(\mathbf{x}_{k}\right)=\max _{\mathbf{x}_{h-1}}\left[s\left(\mathbf{x}_{k}, \mathbf{x}_{k-1}\right)+h_{k-1}\left(\mathbf{x}_{k-1}\right)\right] .
$$

In every successive DP processing stage both the maximizing value of $\mathbf{x}_{k-1}$ and the corresponding maximum value $h_{k-1}$ are chosen. They are stored for each possible value of $\mathbf{x}_{k}$. The a posteriori event probabilities $P_{k \mid k}\left(\mathbf{x}_{k}, \mathbf{x}_{k-1}\right.$, $\left.\ldots, \mathbf{x}_{1} \mid Z_{k}\right)$ and $P_{k \mid k}\left(H_{0} \mid Z_{k}\right)$ are applied to the track sequence and null-target hypotheses, respectively. By applying Bayes Theorem to both the numerator and denominator yields,

$$
\begin{aligned}
& \frac{P_{k \mid k}\left(\mathbf{x}_{k}, \mathbf{x}_{k-1}, \ldots, \mathbf{x}_{1} \mid Z_{k}\right)}{P_{k \mid k}\left(H_{0} \mid Z_{k}\right)} \\
& =\frac{p\left(\mathbf{z}_{k} \mid \mathbf{x}_{k}\right)}{p\left(\mathbf{z}_{k} \mid H_{0}\right)} \cdot \frac{P_{k \mid k-1}\left(\mathbf{x}_{k}, \mathbf{x}_{k-1}, \ldots, \mathbf{x}_{1} \mid Z_{k-1}\right)}{P_{k \mid k-1}\left(H_{0} \mid Z_{k-1}\right)}
\end{aligned}
$$

where $P_{k \mid k-1}$ is the prior probability of the events at the processing state $k$ conditioned on the measurements up to the previous state and $p\left(\mathbf{z}_{k} \mid \mathbf{x}_{k}\right)$ and $p\left(\mathbf{z}_{k} \mid H_{0}\right)$ are the probability density functions of the observations in stage $k$ conditioned on the target-hypothesis and null-hypothesis. The assumption of a first-order Markov model for the target state innovation implies that the state $\mathbf{x}_{k}$ is statistically independent of the previous state $\mathbf{x}_{k-1}$ for the given observations. Hence, the following recursive form is derived by applying the definition of conditional probability to the second term on the right-hand side of the equation (for derivation details refer to [3]).

$$
\begin{aligned}
& \frac{P_{k \mid k}\left(\mathbf{x}_{k}, \mathbf{x}_{k-1}, \ldots, \mathbf{x}_{1} \mid Z_{k}\right)}{P_{k \mid k}\left(H_{0} \mid Z_{k}\right)} \\
& =\frac{p\left(\mathbf{z}_{k} \mid \mathbf{x}_{k}\right)}{p\left(\mathbf{z}_{k} \mid H_{0}\right)} \cdot P\left(\mathbf{x}_{k} \mid \mathbf{x}_{k-1}\right) \cdot \frac{P_{k-1 \mid k-1}\left(\mathbf{x}_{k-1}, \mathbf{x}_{k-2}, \ldots, \mathbf{x}_{1} \mid Z_{k-1}\right)}{P_{k-1 \mid k-1}\left(H_{0} \mid Z_{k-1}\right)}
\end{aligned}
$$

It is usually quite reasonable to assume that given the true state $\mathbf{x}$, the target amplitude is independent of the rate of the target expansion. The target amplitude of the pointlike target depends on the noise sources that are created by the change of weather such as clouds or lighting conditions. 
On the other hand, the rate of expansion is a function of the relative distance between the target and own aircraft [5]. With this assumption, the second term on the right-hand side implies that

$$
\frac{p\left(\mathbf{z}_{k} \mid \mathbf{x}_{k}\right)}{p\left(\mathbf{z}_{k} \mid H_{0}\right)}=\frac{p\left(q_{k} \mid \mathbf{x}_{k}\right)}{p\left(q_{k} \mid H_{0}\right)} \cdot \frac{p\left(r_{k} \mid \mathbf{x}_{k}\right)}{p\left(r_{k} \mid H_{0}\right)}
$$

where $p\left(q_{k} \mid \mathbf{x}_{k}\right), p\left(q_{k} \mid H_{0}\right), p\left(r_{k} \mid \mathbf{x}_{k}\right)$, and $p\left(r_{k} \mid H_{0}\right)$ are the probability density functions of the two independent observations in stage $k$. Finally, the desired DP update equation is given by reinserting the logarithm and carrying out the maximization.

$$
\begin{aligned}
h_{k}\left(\mathbf{x}_{k}\right)=\log & \left(\frac{p\left(q_{k} \mid \mathbf{x}_{k}\right)}{p\left(q_{k} \mid H_{0}\right)}\right)+\log \left(\frac{p\left(r_{k} \mid \mathbf{x}_{k}\right)}{p\left(r_{k} \mid H_{0}\right)}\right) \\
& +\max _{\mathbf{x}_{k-1}}\left[\log P\left(\mathbf{x}_{k} \mid \mathbf{x}_{k-1}\right)+h_{k-1}\left(\mathbf{x}_{k-1}\right)\right] .
\end{aligned}
$$

In the recursive update equation, the function $h_{k}\left(x_{k}\right)$ can be interpreted as the score of the candidate track up to state $\mathbf{x}_{k}$.

\subsection{Implementation of Dynamic Programming}

In order to detect target expansion, a sub-window around each pixel was explored. The sub-image that corresponds to the window is convolved with a smoothing mask. This mask performs low-pass filtering for the output considered as the measure of target strength. This measurement is obtained in terms of the increase of the target strength and it is tracked over a number of stages. The mean rate of expansion was estimated by applying least squares to the target strength.

The probability density functions of the observations are based on the prior knowledge of amplitude and noise characteristics of potential collision threats. These distributions are also adaptively determined from the observation sequence. As the given measurement noise is additive, the likelihood ratio becomes,

$$
\begin{aligned}
& \frac{p\left(q_{k} \mid \mathbf{x}_{k}\right)}{p\left(q_{k} \mid H_{0}\right)}=\frac{p_{t}\left(q_{k}-\hat{q}\right)}{p_{n}\left(q_{k}\right)}, \\
& \frac{p\left(r_{k} \mid \mathbf{x}_{k}\right)}{p\left(r_{k} \mid H_{0}\right)}=\frac{p_{t}\left(r_{k}-\hat{r}\right)}{p_{n}\left(r_{k}\right)}
\end{aligned}
$$

where $p_{t}$ and $p_{n}$ denote the target and noise-only probability density functions, respectively. $\hat{q}$ and $\hat{r}$ are the anticipated target characteristics. In order to model the two output characteristics, a standard Gaussian distribution is developed, whereas the parameters of the distribution are empirically chosen from the data. Other various non-
Gaussian distributions can be used to more accurately reflect on the highly nonlinear characteristics of observations [3].

The transition cost function accounts for the probability of target maneuvers and the noise properties of optical sensors. The transition cost is derived from both a priori models for target maneuvers and the models for state resolution cell quantization and errors in image processing steps. The transition probability is a function of the absolute difference between the expected and actual state at state $k$. Here, the expected state $\mathbf{x}_{k \mid k-1}$ is calculated from the given target model. Thus, the definition of the state transition cost function can be written as follows,

$$
\log P\left(\mathbf{x}_{k} \mid \mathbf{x}_{k-1}\right)=\log P\left(\mathbf{x}_{k}-\mathbf{F}_{k} \mathbf{x}_{k-1}\right) .
$$

As the innovation of the target state is assumed to be independent in time and dimension, the transition costs are separable into the product of transition probabilities of each dimension. These transition probabilities are to be computed from the difference of the Gaussian error functions that are given by,

$$
P(\Delta \mathbf{x})=\operatorname{Erf}\left[\left(\left\lfloor\frac{\Delta \mathbf{x}}{\mathbf{x}_{\text {res }}}\right\rfloor+0.5\right) / \sigma_{\mathbf{x}}\right]-\operatorname{Erf}\left[\left(\left\lfloor\frac{\Delta \mathbf{x}}{\mathbf{x}_{\text {res }}}\right\rfloor-0.5\right) / \sigma_{\mathbf{x}}\right]
$$

where $\Delta \mathbf{x}$ is the difference between the current state and the predicted state, Erf is the Gaussian error function, \ \ is the floor function, $\sigma_{x}$ is the standard deviation of the target innovations in terms of resolution cells and $\mathbf{x}_{\text {res }}$ is the resolution of the quantized states.

\section{Numerical Results}

A DP-based TBD method is based on the proposed track scoring function which is implemented and evaluated by using a synthetic image sequence. In order to carry out the trial our simulation study requires, the image sequence with 100 frames and a frame size of 32 by 32 pixels at 10 frames per second. This is synthetically generated. The image sequence contains the simulation of a moving target that moves through a structured background. A motion effect is caused by jitter and an aperture effect from the optics is also added onto the overall sensor model. In this image sequence, a single artificial target with relatively bright amplitude appears at the 20th frame and it flies from the center to the lower right. The target gradually expanded from the point to the shape of a plus sign that is defined by the image intensity at each pixel position in order to emphasize the target detection capabilities for objects on a collision course.

The results of gray-scale morphological filtering are 


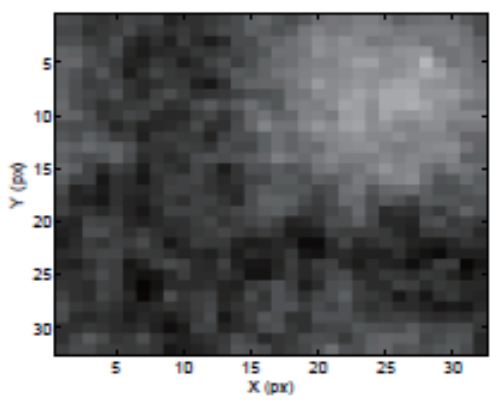

(a)

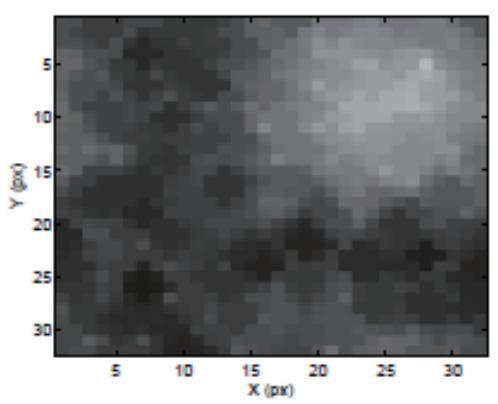

(d)

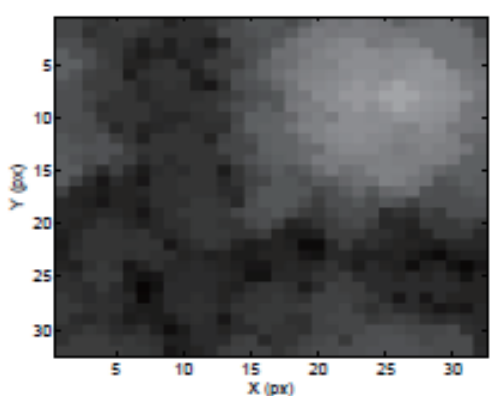

(g)

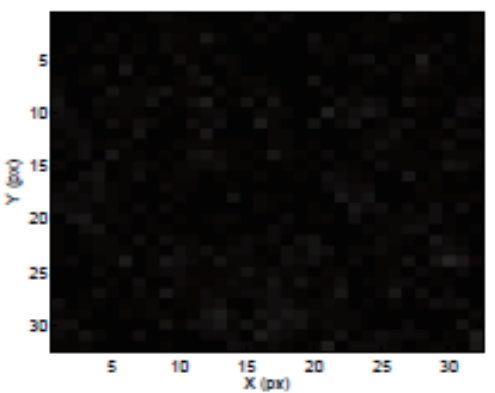

(j)

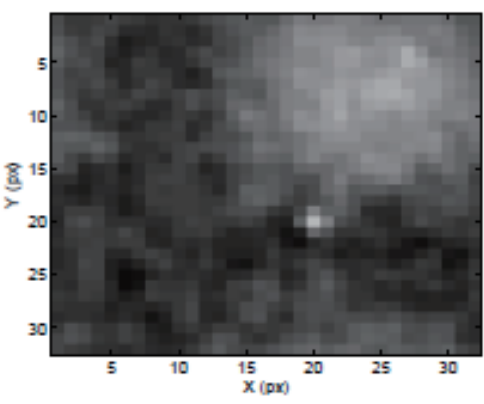

(b)

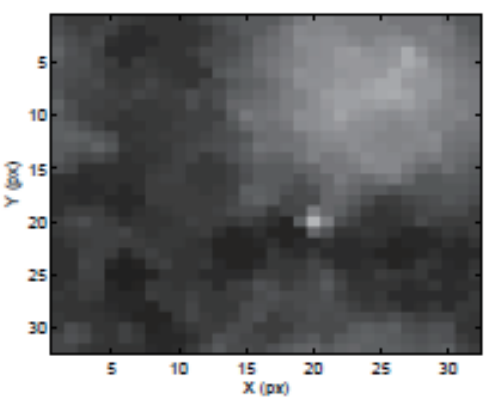

(e)

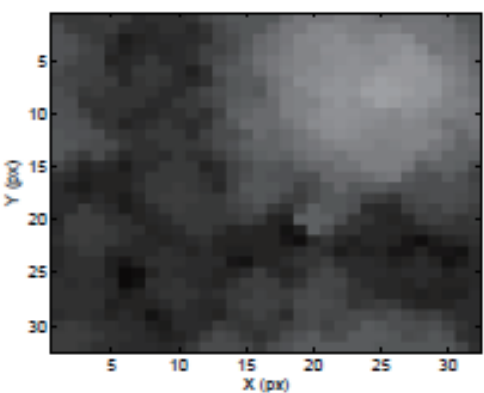

(h)

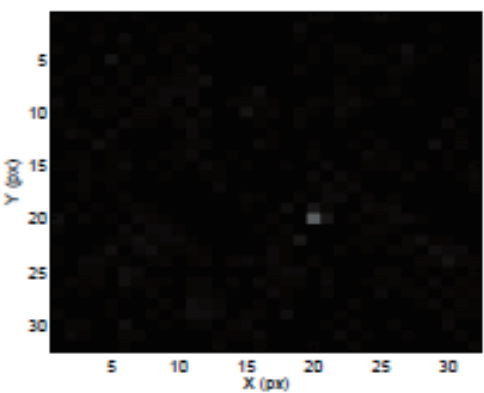

(k)

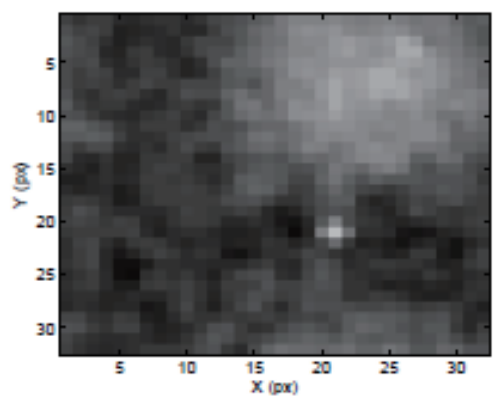

(c)

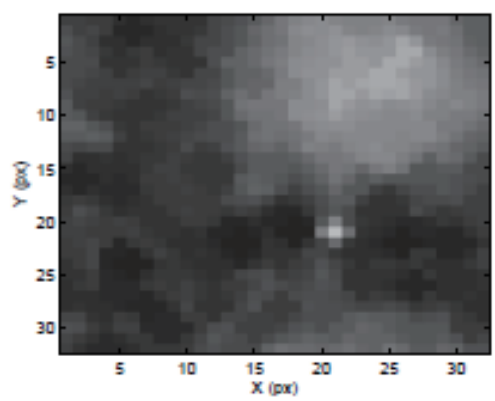

(f)

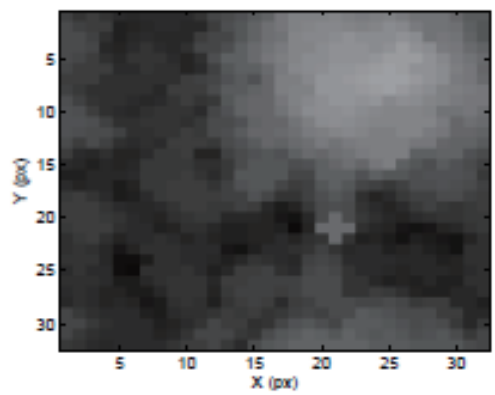

(i)

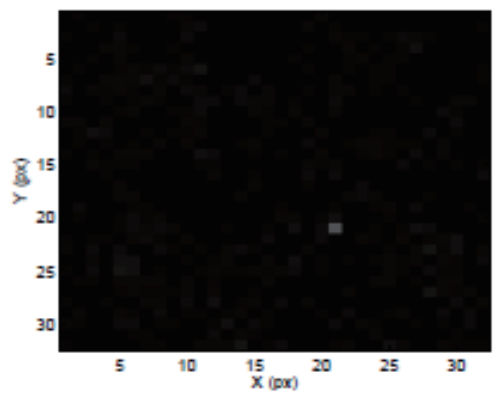

(l)

Fig. 2. Morphological image processing sequence for artificial image data (20th, 40th, and 60th frames for each column). (a)-(c) Raw input images. (d)-(f) Close images. (g)-(i) Open images. (j)-(l) Close-minus-open images. A combination of the two operations extracts the peak regions in images. 
shown in Fig. 2 for the three chosen images. Figures 2.(a)(c) shows the raw input images from the image sequence. Figures 2.(d)-(f) show the results of closing and this tends to preserve small brighter than the background. Figures 2.(g)(i) show the results of an opening and this tends to remove the bright regions that are small in size. Overall image processing results are shown in Figs. 2.(j)-(l). They confirm that the CMO morphological filtering has removed large clutters such as clouds in the background in order to extract point-like targets.

The simulation parameters are given in Table 1. Figure 3 shows the results of the DP algorithm. The displayed intensity value has been scaled to reflect the track scoring for each spatial resolution cell in the chosen velocity plane. The target appears as a bright area which moves near the center of the image in Figs. 3.(b)-(c). The increased signal strength reflects the consistent integration procedure by the DP-based TBD method. This increase in both the size and amplitude of the target is considered in the track scoring function.

Figure 4 shows a comparison of the track scoring functions by taking into account the two different observation sets. The

Table 1. Summary of the simulation parameters

\begin{tabular}{c|c}
\hline DP-based TBD parameters & $\begin{array}{c}\text { Probability distribution } \\
\text { parameters }\end{array}$ \\
\hline$u$ resolution: 1 pix/frame & $\mu_{t}^{q}: 0.5$ \\
$v$ resolution: 1 pix/frame & $\sigma_{t}^{q}: 0.15$ \\
Max. vertical velocity: \pm 1 pix/frame & $\mu_{n}^{q}: 0.2$ \\
Max. horizontal velocity: \pm 1 pix/frame & $\sigma_{n}^{q}: 0.2$ \\
$x$ search window: \pm 3 pixels & $\mu_{t}^{r}: 0.1 \mathrm{pix}^{2} /$ frame \\
$y$ search window: \pm 3 pixels & $\sigma_{t}^{r}: 0.08 \mathrm{pix}^{2} /$ frame \\
$u$ search window: \pm 1 pixels/frame & $\mu_{n}^{r}:-0.1 \mathrm{pix}^{2} /$ frame \\
$v$ search window: \pm 1 pixels/frame & $\sigma_{n}^{r}: 0.1 \mathrm{pix}^{2} /$ frame \\
\hline
\end{tabular}

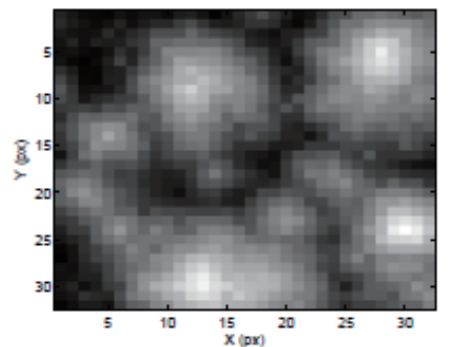

(a)

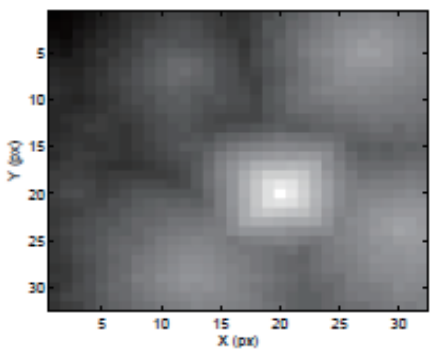

(b)

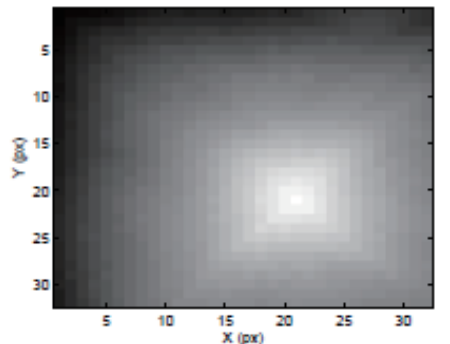

(c)

Fig. 3. The DP-TBD method applied on the image sequence with the proposed track scoring function: (a)-(c) Three frames from sequence (20th, 40th, and 60th frames). As the algorithm accumulates scores by evaluating the target's amplitude and expansion rate, the existence and the state of the target are revealed more clearly.

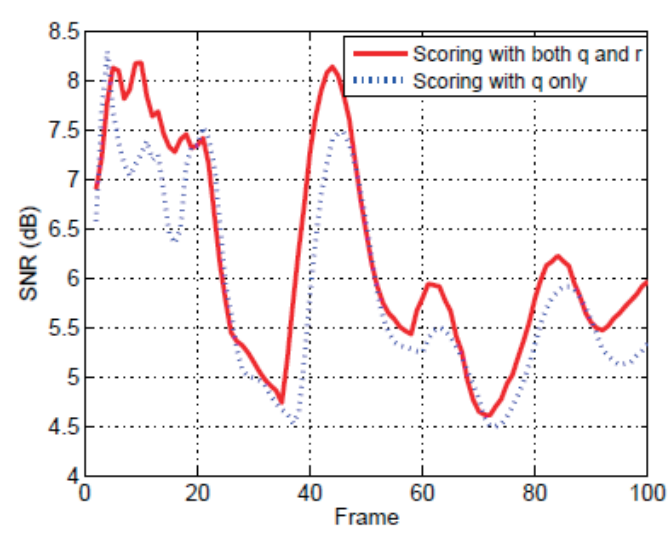

(a)
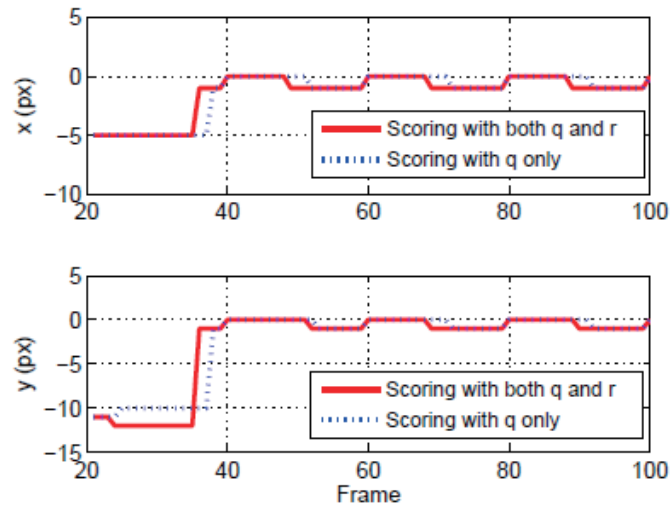

(b)

Fig. 4. Comparison of two track scoring functions from different observation sets: the solid line is the result calculated from the two observations and the dotted line is that from the amplitude observation only. (a) Signal-to-noise ratio. (b) Detection errors between the true target positions and the estimated position of maximum scores. 
first observation set provides both $q$ and $r$ observations to the DP module. The second observation set consists of only the target amplitude estimates. In each stage, the pixel that has the maximum value from DP-based track scoring functions is considered as a potential target without a decision step. As expected, Fig. 4.(a) indicates that the detection performance of the DP-based TBD method with the proposed track scoring function is slightly improved in terms of the SNR. The position error depicted in Fig. 4.(b) shows that it converges a number of frames that are advanced as two collision properties which are considered in the track scoring function.

\section{Conclusion}

In this paper, a track scoring function of DP-based TBD method is proposed to take account of the two distinguishable properties of airborne targets on a near-collision course. The findings from the numerical results indicate that the signalto-ratio with the modified track scoring function is slightly improved compared to the previous one. Further work is being undertaken and it includes the implementation of the DP-based TBD method applied for airborne target detection.

\section{Acknowledgements}

This research was sponsored by the Korea Aerospace Research Institute (KARI) under the Vision-Based Collision Avoidance Techniques for UAVs Project.

\section{References}

[1] Barniv, Y., "Dynamic programming solution for detecting dim moving targets", IEEE Transactions on Aerospace and Electronic Systems, Vol. AES-21, Issue 1, 1985, pp. 144-156.

[2] Barniv, Y., and Kella, O., "Dynamic programming solution for detecting dim moving targets part II: analysis", IEEE Transactions on Aerospace and Electronic Systems, Vol. AES-23, Issue 6, 1987, pp. 776-788.

[3] Arnold, J., Shaw, S. W., and Pasternack, H., "Efficient target tracking using dynamic programming", IEEE Transactions on Aerospace and Electronic Systems, Vol. 29, Issue 1, 1993, pp. 44-56.

[4] Tonissen, S. M., and Evans, R. J., "Performance of dynamic programming techniques for Track-Before-Detect", IEEE Transactions on Aerospace and Electronic Systems, Vol. 32, Issue 4, 1996, pp. 1440-1451.

[5] Blackman, S., and Popoli, R., Design and analysis of modern tracking systems, Artech House Norwood, MA, 1999.

[6] Johnston, L. A., and Krishnamurthy, V., "Performance analysis of a dynamic programming track before detect algorithm", IEEE Transactions on Aerospace and Electronic Systems, Vol. 38, Issue 1, 2002, pp. 228-242.

[7] Nichtern, O., and Rotman, S., "Parameter Adjustment for a Dynamic Programming Track-before-Detect-Based Target Detection Algorithm", EURASIP Journal on Advances in Signal Processing, Vol. 2008, 2008, pp. 1-19.

[8] Gandhi, T., Yang, M., Kasturi, R., Camps, O., Coraor, L., and McCandless, J., "Detection of obstacles in the flight path of an aircraft", IEEE Transactions on Aerospace and Electronic Systems, Vol. 39, Issue 1, 2003, pp. 176-191.

[9] Carnie, R., Walker, R., and Corke, P., "Image processing algorithms for UAV Sense and Avoid", Proceedings of the 2006 IEEE International Conference on Robotics and Automation, Orlando, FL, 2006. 